\title{
The spectrum of simplicial volume of non-compact manifolds
}

\author{
Nicolaus Heuer ${ }^{1}$. Clara Löh ${ }^{2}$ \\ Received: 5 November 2020 / Accepted: 10 August 2021 / Published online: 3 September 2021 \\ (c) The Author(s) 2021
}

\begin{abstract}
We show that, in dimension at least 4 , the set of locally finite simplicial volumes of oriented connected open manifolds is $[0, \infty]$. Moreover, we consider the case of tame open manifolds and some low-dimensional examples.
\end{abstract}

Keywords Simplicial volume $\cdot$ Non-compact manifolds

Mathematics Subject Classification 57N65

\section{Introduction}

Simplicial volumes are invariants of manifolds defined in terms of the $\ell^{1}$-semi-norm on singular homology [9].

Definition 1.1 (simplicial volume) Let $M$ be an oriented connected $d$-manifold without boundary. Then the simplicial volume of $M$ is defined by

$$
\|M\|^{\text {lf }}:=\inf \left\{|c|_{1} \mid c \in C_{d}^{\text {lf }}(M ; \mathbb{R}) \text { is a fundamental cycle of } M\right\},
$$

where $C_{*}^{\text {lf }}$ denotes the locally finite singular chain complex. If $M$ is compact, then we also write $\|M\|:=\|M\|^{\text {lf }}$. Using relative fundamental cycles, the notion of simplicial volume can be extended to oriented manifolds with boundary.

Simplicial volumes are related to negative curvature, volume estimates, and amenability [9]. In the present article, we focus on simplicial volumes of non-compact manifolds. Only few concrete results are known in this context: There are computations for certain locally symmetric spaces $[3,12,15,16]$ as well as the general volume estimates [9], vanishing results $[8,9]$, and finiteness results $[9,14]$.

$\triangle$ Clara Löh

clara.loeh@mathematik.uni-r.de

http://www.mathematik.uni-r.de/loeh

Nicolaus Heuer

nh441@cam.ac.uk

https://www.dpmms.cam.ac.uk/ nh441

1 DPMMS, University of Cambridge, Cambridge, UK

2 Fakultät für Mathematik, Universität Regensburg, 93040 Regensburg, Germany 
Let $d \in \mathbb{N}$, let $M(d)$ be the class of all oriented closed connected $d$-manifolds, and let $M^{\mathrm{lf}}(d)$ be the class of all oriented connected manifolds without boundary. Then we set $\mathrm{SV}(d):=\{\|M\| \mid M \in M(d)\}$ and

$$
\operatorname{SV}^{\mathrm{lf}}(d):=\left\{\|M\|^{\mathrm{lf}} \mid M \in M^{\mathrm{lf}}(d)\right\} .
$$

It is known that $\mathrm{SV}(d)$ is countable and that this set has no gap at 0 if $d \geq 4$ :

Theorem $1.2\left[10\right.$, Theorem A] Let $d \in \mathbb{N}_{\geq 4}$. Then $\mathrm{SV}(d)$ is dense in $\mathbb{R}_{\geq 0}$ and $0 \in \mathrm{SV}(d)$.

In contrast, if we allow non-compact manifolds, we can realise all non-negative real numbers:

Theorem A Let $d \in \mathbb{N}_{\geq 4}$. Then $\operatorname{SV}^{\mathrm{lf}}(d)=[0, \infty]$.

The proof uses the no-gap theorem Theorem 1.2 and a suitable connected sum construction.

If we restrict to tame manifolds, then we are in a similar situation as in the closed case:

Theorem B Let $d \in \mathbb{N}$. Then the set $\mathrm{SV}_{\text {tame }}^{\mathrm{lf}}(d) \subset[0, \infty]$ is countable. In particular, the set $[0, \infty] \backslash \mathrm{SV}_{\text {tame }}^{\mathrm{lf}}(d)$ is uncountable.

As an explicit example, we compute $S^{l f}(2)$ and $S_{\text {tame }}^{\text {lf }}$ (2) (Proposition 4.2) as well as $\mathrm{SV}_{\text {tame }}^{\mathrm{lf}}$ (3) (Proposition 4.3). The case of non-tame 3-manifolds seems to be fairly tricky.

Question 1.3 What is $\mathrm{SV}^{\mathrm{lf}}(3)$ ?

As $S V(4) \subset S V_{\text {tame }}^{\text {lf }}(4)$, we know that $S V_{\text {tame }}^{\text {lf }}$ (4) contains arbitrarily small transcendental numbers [11].

From a geometric point of view, the so-called Lipschitz simplicial volume is more suitable for Riemannian non-compact manifolds than the locally finite simplicial volume. It is therefore natural to ask the following:

Question 1.4 Do Theorem A and Theorem B also hold for the Lipschitz simplicial volume of oriented connected open Riemannian manifolds?

\section{Organisation of this article}

Section 2 contains the proof of Theorem A. The proof of Theorem B is given in Sect. 3. The low-dimensional case is treated in Sect. 4.

\section{Proof of Theorem A}

Let $d \in \mathbb{N}_{\geq 4}$ and let $\alpha \in[0, \infty]$. Because $\mathrm{SV}(d)$ is dense in $\mathbb{R}_{\geq 0}$ (Theorem 1.2), there exists a sequence $\left(\alpha_{n}\right)_{n \in \mathbb{N}}$ in $\operatorname{SV}(d)$ with $\sum_{n=0}^{\infty} \alpha_{n}=\alpha$.

\subsection{Construction}

We first describe the construction of a corresponding oriented connected open manifold $M$ : For each $n \in \mathbb{N}$, we choose an oriented closed connected $d$-manifold $M_{n}$ with $\left\|M_{n}\right\|=\alpha_{n}$. Moreover, for $n>0$, we set

$$
W_{n}:=M_{n} \backslash\left(B_{n,-}^{\circ} \sqcup B_{n,+}^{\circ}\right),
$$




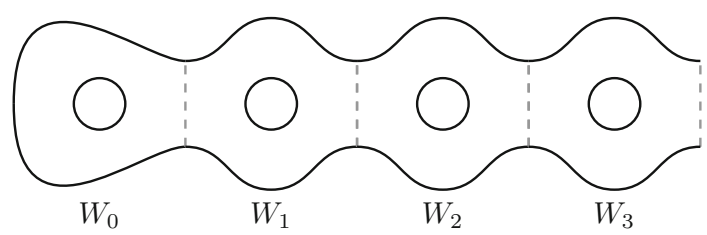

Fig. 1 The construction of $M$ for the proof of Theorem A

where $B_{n,-}=i_{n,-}\left(D^{d}\right)$ and $B_{n,+}=i_{n,+}\left(D^{d}\right)$ are two disjointly embedded closed $d$-balls in $M_{n}$. Similarly, we set $W_{0}:=M_{0} \backslash B_{0,+}^{\circ}$. Furthermore, we choose an orientation-reversing homeomorphism $f_{n}: S^{d-1} \rightarrow S^{d-1}$. We then consider the infinite "linear" connected sum manifold (Fig. 1)

$$
\begin{aligned}
M & :=M_{0} \# M_{1} \# M_{2} \# \ldots \\
& =\left(W_{0} \sqcup W_{1} \sqcup W_{n} \sqcup \ldots\right) / \sim,
\end{aligned}
$$

where $\sim$ is the equivalence relation generated by

$$
i_{n+1,-}(x) \sim i_{n,+}\left(f_{n}(x)\right)
$$

for all $n \in \mathbb{N}$ and all $x \in S^{d-1} \subset D^{d}$; we denote the induced inclusion $W_{n} \rightarrow M$ by $i_{n}$. By construction, $M$ is connected and inherits an orientation from the $M_{n}$.

\subsection{Computation of the simplicial volume}

We will now verify that $\|M\|^{\text {lf }}=\alpha$ :

Claim 2.1 We have $\|M\|^{\text {lf }} \leq \alpha$.

Proof The proof is a straightforward adaption of the chain-level proof of sub-additivity of simplicial volume with respect to amenable glueings.

In particular, we will use the uniform boundary condition [19] and the equivalence theorem [2,9]:

UBC The chain complex $C_{*}\left(S^{d-1} ; \mathbb{R}\right)$ satisfies $(d-1)$-UBC, i.e., there is a constant $K$ such that: For each $c \in \operatorname{im} \partial_{d} \subset C_{d-1}\left(S^{d-1} ; \mathbb{R}\right)$, there exists a chain $b \in C_{d}\left(S^{d-1} ; \mathbb{R}\right)$ with

$$
\partial_{d} b=c \text { and }|b|_{1} \leq K \cdot|c|_{1} .
$$

EQT Let $N$ be an oriented closed connected $d$-manifold, let $B_{1}, \ldots, B_{k}$ be disjointly embedded $d$-balls in $N$, and let $W:=N \backslash\left(B_{1}^{\circ} \cup \ldots, \cup B_{k}^{\circ}\right)$. Moreover, let $\in \in \mathbb{R}_{>0}$. Then

$$
\|N\|=\inf \left\{\left.|z|_{1}|z \in Z(W ; \mathbb{R}),| \partial_{d} z\right|_{1} \leq \epsilon\right\},
$$

where $Z(W ; \mathbb{R}) \subset C_{d}(W ; \mathbb{R})$ denotes the set of all relative fundamental cycles of $W$.

Let $\in \in \mathbb{R}_{>0}$. By EQT, for each $n \in \mathbb{N}$, there exists a relative fundamental cycle $z_{n} \in$ $Z\left(W_{n} ; \mathbb{R}\right)$ with

$$
\left|z_{n}\right|_{1} \leq \alpha_{n}+\frac{1}{2^{n}} \cdot \epsilon \text { and }\left|\partial_{d} z_{n}\right|_{1} \leq \frac{1}{2^{n}} \cdot \epsilon
$$


We now use UBC to construct a locally finite fundamental cycle of $M$ out of these relative cycles: For $n \in \mathbb{N}$, the boundary parts $C_{d-1}\left(i_{n} ; \mathbb{R}\right)\left(\left.\partial_{d} z_{n}\right|_{B_{n,+}}\right)$ and $-C_{d-1}\left(i_{n+1} ; \mathbb{R}\right)\left(\left.\partial_{d} z_{n+1}\right|_{B_{n+1},-}\right)$ are fundamental cycles of the sphere $S^{d-1}$ (embedded via $i_{n} \circ i_{n,+}$ and $i_{n+1} \circ i_{n+1,-}$ into $M$, which implicitly uses the orientation-reversing homeomorphism $\left.f_{n}\right)$. By UBC, there exists a chain $b_{n} \in C_{d}\left(S^{d-1} ; \mathbb{R}\right)$ with

$$
\begin{aligned}
\partial_{d} C_{d}\left(i_{n} \circ i_{n,+} ; \mathbb{R}\right)\left(b_{n}\right)= & C_{d-1}\left(i_{n} ; \mathbb{R}\right)\left(\left.\partial_{d} z_{n}\right|_{B_{n,+}}\right) \\
& +C_{d-1}\left(i_{n+1} ; \mathbb{R}\right)\left(\left.\partial_{d} z_{n+1}\right|_{B_{n+1,-}}\right)
\end{aligned}
$$

and

$$
\left|b_{n}\right|_{1} \leq K \cdot\left(\frac{1}{2^{n}}+\frac{1}{2^{n+1}}\right) \cdot \epsilon \leq K \cdot \frac{1}{2^{n-1}} \cdot \epsilon .
$$

A straightforward computation shows that

$$
c:=\sum_{n=0}^{\infty} C_{d}\left(i_{n} ; \mathbb{R}\right)\left(z_{n}-C_{d}\left(i_{n,+} ; \mathbb{R}\right)\left(b_{n}\right)\right)
$$

is a locally finite $d$-cycle on $M$. Moreover, the local contribution on $W_{0}$ shows that $c$ is a locally finite fundamental cycle of $M$. By construction,

$$
\begin{aligned}
|c|_{1} & \leq \sum_{n=0}^{\infty}\left(\left|z_{n}\right|_{1}+\left|b_{n}\right|_{1}\right) \\
& \leq \sum_{n=0}^{\infty}\left(\alpha_{n}+\frac{1}{2^{n}} \cdot \epsilon+K \cdot \frac{1}{2^{n-1}} \cdot \epsilon\right) \leq \sum_{n=0}^{\infty} \alpha_{n}+(2+4 \cdot K) \cdot \epsilon \\
& =\alpha+(2+4 \cdot K) \cdot \epsilon .
\end{aligned}
$$

Thus, taking $\epsilon \rightarrow 0$, we obtain $\|M\|^{\text {lf }} \leq \alpha$.

Claim 2.2 We have $\|M\|^{\text {lf }} \geq \alpha$.

Proof Without loss of generality we may assume that $\|M\|^{\mathrm{lf}}$ is finite. Let $c \in C_{d}^{\mathrm{lf}}(M ; \mathbb{R})$ be a locally finite fundamental cycle of $M$ with $|c|_{1}<\infty$. For $n \in \mathbb{N}$, we consider the subchain $c_{n}:=\left.c\right|_{W_{(n)}}$ of $c$, consisting of all simplices whose images touch $W_{(n)}:=$ $\bigcup_{k=0}^{n} i_{k}\left(W_{k}\right) \subset M$. Because $c$ is locally finite, each $c_{n}$ is a finite singular chain and $\left(\left|c_{n}\right|_{1}\right)_{n \in \mathbb{N}}$ is a monotonically increasing sequence with limit $|c|_{1}$.

Let $\epsilon \in \mathbb{R}_{>0}$. Then there is an $n \in \mathbb{N}_{>0}$ that satisfies $\left|c-c_{n}\right|_{1} \leq \epsilon$ and $\alpha-\sum_{k=0}^{n} \alpha_{k} \leq \epsilon$. Let

$$
p: M \rightarrow W_{(n)} / i_{n}\left(B_{n,+}\right)=: W
$$

be the map that collapses everything beyond stage $n+1$ to a single point $x$. Then $z:=$ $C_{d}(p ; \mathbb{R})\left(c_{n}\right) \in C_{d}(W,\{x\} ; \mathbb{R})$ is a relative cycle and

$$
\left|\partial_{d} z\right|_{1} \leq\left|\partial_{d} c_{n}\right|_{1} \leq\left|\partial_{d}\left(c-c_{n}\right)\right|_{1} \leq(d+1) \cdot\left|c-c_{n}\right|_{1} \leq(d+1) \cdot \epsilon .
$$

Because $d>1$, there exists a chain $b \in C_{d}(\{x\} ; \mathbb{R})$ with

$$
\partial_{d} b=\partial_{d} z \text { and }|b|_{1} \leq\left|\partial_{d} z\right| \leq(d+1) \cdot \epsilon .
$$

Then

$$
\bar{z}:=z-b \in C_{d}(W ; \mathbb{R})
$$


is a cycle on $W$; because $z$ and $\bar{z}$ have the same local contribution on $W_{0}$, the cycle $z$ is a fundamental cycle of the manifold

$$
W \cong M_{0} \# \ldots \# M_{n} .
$$

As $d>2$, the construction of our chains and additivity of simplicial volume under connected sums [2,9] show that

$$
\begin{aligned}
|c|_{1} & \geq\left|c_{n}\right|_{1} \geq|z|_{1} \geq|\bar{z}|_{1}-|b|_{1} \\
& \geq\|W\|-(d+1) \cdot \epsilon=\sum_{k=0}^{n}\left\|M_{k}\right\|-(d+1) \cdot \epsilon \\
& \geq \alpha-(d+2) \cdot \epsilon .
\end{aligned}
$$

Thus, taking $\epsilon \rightarrow 0$, we obtain $|c|_{1} \geq \alpha$; hence, $\|M\|^{\text {lf }} \geq \alpha$.

This completes the proof of Theorem A.

Remark 2.3 (adding geometric structures) In fact, this argument can also be performed smoothly: The constructions leading to Theorem 1.2 can be carried out in the smooth setting. Therefore, we can choose the $\left(M_{n}\right)_{n \in \mathbb{N}}$ to be smooth and equip $M$ with a corresponding smooth structure. Moreover, we can endow these smooth pieces with Riemannian metrics. Scaling these Riemannian metrics appropriately shows that we can turn $M$ into a Riemannian manifold of finite volume.

\section{Proof of Theorem B}

In this section, we prove Theorem B, i.e., that the set of simplicial volumes of tame manifolds is countable.

Definition 3.1 A manifold $M$ without boundary is tame if there exists a compact connected manifold $W$ with boundary such that $M$ is homeormorphic to $W^{\circ}:=W \backslash \partial W$.

As in the closed case, our proof is based on a counting argument:

Proposition 3.2 There are only countably many proper homotopy types of tame manifolds.

As we could not find a proof of this statement in the literature, we will give a complete proof in Sect. 3.1 below. Theorem B is a direct consequence of Proposition 3.2:

Proof of Theorem $B$ The simplicial volume $\|\cdot\|^{\text {lf }}$ is invariant under proper homotopy equivalence (this can be shown as in the compact case). Therefore, the countability of $\operatorname{SV}^{\mathrm{lf}}(d)$ follows from the countability of the set of proper homotopy types of tame $d$-manifolds (Proposition 3.2).

Remark 3.3 Let $d \in \mathbb{N}_{\geq 3}$. Then $\infty \in \mathrm{SV}_{\text {tame }}^{\text {lf }}(d)$ : Let $N$ be an oriented closed connected hyperbolic $(d-1)$-manifold and let $M:=N \times \mathbb{R}$. Then $M$ is tame (as interior of $N \times[0,1])$ and $\|N\|>0$ [9, Section 0.3] [23, Theorem 6.2]. Hence, by the finiteness criterion [9, p. 17] $\left[14\right.$, Theorem 6.4], we obtain that $\|M\|^{\text {lf }}=\infty$. 


\subsection{Counting tame manifolds}

It remains to prove Proposition 3.2. We use the following observations:

Definition 3.4 (models of tame manifolds)

- A model of a tame manifold $M$ is a finite CW-pair $(X, A)$ (i.e., a finite CW-complex $X$ with a finite subcomplex $A$ ) that is homotopy equivalent (as pairs of spaces) to ( $W, \partial W)$, where $W$ is a compact connected manifold with boundary whose interior is homeomorphic to $M$.

- Two models of tame manifolds are equivalent if they are homotopy equivalent as pairs of spaces.

Lemma 3.5 (existence of models) Let $W$ be a compact connected manifold. Then there exists a finite $C W$-pair $(X, A)$ such that $(W, \partial W)$ and $(X, A)$ are homotopy equivalent pairs of spaces.

In particular: Every tame manifold admits a model.

Proof It should be noted that we work with topological manifolds; hence, we cannot argue directly via triangulations. Of course, the main ingredient is the fact that every compact manifold is homotopy equivalent to a finite complex $[13,22]$.

Hence, there exist finite CW-complexes $A$ and $Y$ with homotopy equivalences $f: A \rightarrow$ $\partial W$ and $g: Y \rightarrow W$. Let $j:=\bar{g} \circ i \circ f$, where $i: \partial W \hookrightarrow W$ is the inclusion and $\bar{g}$ is a homotopy inverse of $g$. By construction, the upper square in the diagram in Fig. 2 is homotopy commutative.

As next step, we replace $j: A \rightarrow Y$ by a homotopic map $j_{c}: A \rightarrow Y$ that is cellular (second square in Fig. 2).

The mapping cylinder $Z$ of $j_{c}$ has a finite $\mathrm{CW}$-structure (as $j_{c}$ is cellular) and the canonical map $p: Z \rightarrow Y$ allows to factor $j_{c}$ into an inclusion $J$ of a subcomplex and the homotopy equivalence $p$ (third square in Fig. 2).

We thus obtain a homotopy commutative square

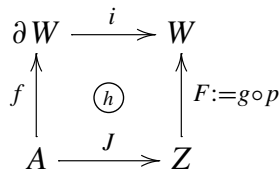

where the vertical arrows are homotopy equivalences, the upper horizontal arrow is the inclusion, and the lower horizontal arrow is the inclusion of a subcomplex.

Using a homotopy between $i \circ f$ and $F \circ J$ and adding another cylinder to $Z$, we can replace $Z$ by a finite $C W$-complex $X$ (that still contains $A$ as subcomplex) to obtain a strictly commutative diagram

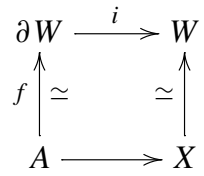

whose vertical arrows are homotopy equivalences and whose horizontal arrows are inclusions.

Because the inclusions $\partial W \hookrightarrow W$ (as inclusion of the boundary of a compact topological manifold) and $A \hookrightarrow X$ (as inclusion of a subcomplex) are cofibrations, this already implies that the vertical arrows form a homotopy equivalence $(X, A) \rightarrow(W, \partial W)$ of pairs $[18$, Chapter 6.5]. 


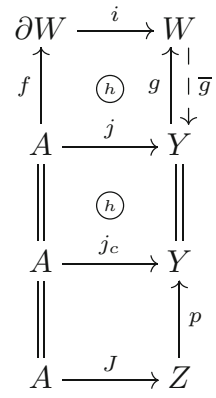

Fig. 2 Finding a model

Lemma 3.6 (equivalence of models) If $M$ and $N$ are tame manifolds with equivalent models, then $M$ and $N$ are properly homotopy equivalent.

Proof As $M$ and $N$ admit equivalent models, there exist compact connected manifolds $W$ and $V$ with boundary such that $M \cong W^{\circ}$ and $N \cong V^{\circ}$ and such that the pairs $(W, \partial W)$ and $(V, \partial V)$ are homotopy equivalent (by transitivity of homotopy equivalence of pairs of spaces $)$. Let $\left(f, f_{\partial}\right):(W, \partial W) \rightarrow(V, \partial V)$ and $\left(g, g_{\partial}\right):(V, \partial V) \rightarrow(W, \partial W)$ be mutually homotopy inverse homotopy equivalences of pairs.

By the topological collar theorem [5,6], we have homeomorphisms

$$
\begin{aligned}
& M \cong W \cup_{\partial W}(\partial W \times[0, \infty)) \\
& N \cong V \cup_{\partial V}(\partial V \times[0, \infty)),
\end{aligned}
$$

where the glueing occurs via the canonical inclusions $\partial W \hookrightarrow \partial W \times[0, \infty)$ and $\partial V \hookrightarrow$ $\partial V \times[0, \infty)$ at parameter 0 .

Then the maps $f$ and $f_{\partial} \times \operatorname{id}_{[0, \infty)}$ glue to a well-defined proper continuous map $F: M \rightarrow$ $N$ and the maps $g$ and $g_{\partial} \times \operatorname{id}_{[0, \infty)}$ glue to a well-defined proper continuous map $G: N \rightarrow M$.

Moreover, the homotopy of pairs between $\left(f \circ g, f_{\partial} \circ g_{\partial}\right)$ and $\left(\mathrm{id}_{V}, \mathrm{id}_{\partial V}\right)$ glues into a proper homotopy between $F \circ G$ and $\operatorname{id}_{M}$. In the same way, there is a proper homotopy between $G \circ F$ and $\operatorname{id}_{N}$. Hence, the spaces $M$ and $N$ are properly homotopy equivalent.

Lemma 3.7 (countability of models) There exist only countably many equivalence classes of models.

Proof There are only countably many homotopy types of finite CW-complexes (because every finite $\mathrm{CW}$-complex is homotopy equivalent to a finite simplicial complex). Moreover, every finite $\mathrm{CW}$-complex has only finitely many subcomplexes. Therefore, there are only countably many homotopy types (of pairs of spaces) of finite CW-pairs.

Proof of Proposition 3.2 We only need to combine Lemma 3.5, Lemma 3.6, and Lemma 3.7.

\section{Low dimensions}

\subsection{Dimension 2}

We now compute the set of simplicial volumes of surfaces. We first consider the tame case: 
Example 4.1 (tame surfaces) Let $W$ be an oriented compact connected surface with $g \in \mathbb{N}$ handles and $b \in \mathbb{N}$ boundary components. Then the proportionality principle for simplicial volume of hyperbolic manifolds [9, p. 11] (a thorough exposition is given, for instance, by Fujiwara and Manning [7, Appendix A]) gives

$$
\left\|W^{\circ}\right\|^{\text {lf }}= \begin{cases}4 \cdot(g-1)+2 \cdot b & \text { if } g>0 \\ 2 \cdot b-4 & \text { if } g=0 \text { and } b>1 \\ 0 & \text { if } g=0 \text { and } b \in\{0,1\} .\end{cases}
$$

Proposition 4.2 We have $\mathrm{SV}^{\mathrm{lf}}(2)=2 \cdot \mathbb{N} \cup\{\infty\}$ and $\mathrm{SV}_{\text {tame }}^{\mathrm{lf}}(2)=2 \cdot \mathbb{N}$.

Proof We first prove $2 \cdot \mathbb{N} \subset \mathrm{SV}_{\text {tame }}^{\text {lf }}$ (2) $\subset \mathrm{SV}^{\mathrm{lf}}(2)$ and $\infty \in \mathrm{SV}^{\mathrm{lf}}(2)$, i.e., that all the given values may be realised: In view of Example 4.1, all even numbers occur as simplicial volume of some (possibly open) tame surface.

Let

$$
M:=T^{2} \# T^{2} \# T^{2} \# \ldots
$$

be an infinite "linear" connected sum of tori $T^{2}$. Collapsing $M$ to the first $g \in \mathbb{N}$ summands and an argument as in the proof of Claim 2.2 shows that

$$
\|M\|^{\text {lf }} \geq\left\|\Sigma_{g}\right\|=4 \cdot g-4
$$

for all $g \in \mathbb{N}_{\geq 1}$. Hence, $\|M\|^{\text {lf }}=\infty$.

It remains to show that $\mathrm{SV}^{\mathrm{lf}}(2) \subset 2 \cdot \mathbb{N} \cup\{\infty\}$ : Let $M$ be an oriented connected (topological, separable, Hausdorff) 2-manifold without boundary. Then $M$ admits a smooth structure [20] and whence a proper smooth map $p: M \rightarrow \mathbb{R}$. Using suitable regular values of $p$, we can thus write $M$ as an ascending union

$$
M=\bigcup_{n \in \mathbb{N}} M_{n}
$$

of oriented connected compact submanifolds (possibly with boundary) $M_{n}$ that are nested via $M_{0} \subset M_{1} \subset \ldots$ Then one of the following cases occurs:

1. There exists an $N \in \mathbb{N}$ such that for all $n \in \mathbb{N}_{\geq N}$ the inclusion $M_{n} \hookrightarrow M_{n+1}$ is a homotopy equivalence.

2. For each $N \in \mathbb{N}$ there exists an $n \in \mathbb{N}_{\geq N}$ such that the inclusion $M_{n} \hookrightarrow M_{n+1}$ is not a homotopy equivalence.

In the first case, the classification of compact surfaces with boundary shows that $M$ is tame. Hence $\|M\|^{\text {lf }} \in 2 \cdot \mathbb{N}$ (Example 4.1).

In the second case, the manifold $M$ is not tame (which can, e.g., be derived from the classification of compact surfaces with boundary). We show that $\|M\|^{\mathrm{lf}}=\infty$. To this end. we distinguish two cases:

a. The sequence $\left(h\left(M_{n}\right)\right)_{n \in \mathbb{N}}$ is unbounded, where $h(\cdot)$ denotes the number of handles of the surface.

b. The sequence $\left(h\left(M_{n}\right)\right)_{n \in \mathbb{N}}$ is bounded.

In the unbounded case, a collapsing argument (similar to the argument for $T^{2} \# T^{2} \# \ldots$ and Claim 2.2) shows that $\|M\|^{\text {lf }}=\infty$.

We claim that also in the bounded case we have $\|M\|^{\text {lf }}=\infty$ : Shifting the sequence in such a way that all handles are collected in $M_{0}$, we may assume without loss of generality that 
the sequence $\left(h\left(M_{n}\right)\right)_{n \in \mathbb{N}}$ is constant. Thus, for each $n \in \mathbb{N}$, the surface $M_{n+1}$ is obtained from $M_{n}$ by adding a finite disjoint union of disks and of spheres with finitely many (at least two) disks removed; we can reorganise this sequence in such a way that no disks are added. Hence, we may assume that $M_{n}$ is a retract of $M_{n+1}$ for each $n \in \mathbb{N}$. Furthermore, because we are in case 2, the classification of compact surfaces shows (with the help of Example 4.1) that

$$
\lim _{n \rightarrow \infty}\left\|M_{n}\right\|=\infty
$$

Let $c \in C_{2}^{\mathrm{lf}}(M ; \mathbb{R})$ be a locally finite fundamental cycle of $M$ and let $n \in \mathbb{N}$. Because $c$ is locally finite, there is a $k \in \mathbb{N}$ such that $\left.c\right|_{M_{n}}$ is supported on $M_{n+k}$; the restriction $\left.c\right|_{M_{n}}$ consists of all summands of $c$ whose supports intersect with $M_{n}$. Because $M_{n}$ is a retract of $M_{n+k}$, we obtain from $\left.c\right|_{M_{n}}$ a relative fundamental cycle $c_{n}$ of $M_{n}$ by pushing the chain $\left.c\right|_{M_{n}}$ to $M_{n}$ via a retraction $M_{n+k} \rightarrow M_{n}$. Therefore,

$$
|c|_{1} \geq\left.|c|_{M_{n}}\right|_{1} \geq\left|c_{n}\right|_{1} \geq\left\|M_{n}\right\| .
$$

Taking $n \rightarrow \infty$ shows that $|c|_{1}=\infty$. Taking the infimum over all locally finite fundamental cycles $c$ of $M$ proves that $\|M\|^{\text {lf }}=\infty$.

Moreover, Example 4.1 shows that $\infty \notin \mathrm{SV}_{\text {tame }}^{\text {lf }}$ (2).

\subsection{Dimension 3}

The general case of non-compact 3-manifolds seems to be rather involved (as the structure of non-compact 3-manifolds can get fairly complicated). We can at least deal with the tame case:

Proposition 4.3 We have $\mathrm{SV}_{\text {tame }}^{\mathrm{lf}}(3)=\mathrm{SV}(3) \cup\{\infty\}$.

Proof Clearly, SV(3) $\subset \mathrm{SV}_{\text {tame }}^{\mathrm{lf}}$ (3) and $\infty \in \mathrm{SV}_{\text {tame }}^{\mathrm{lf}}$ (3) (Remark 3.3).

Conversely, let $W$ be an oriented compact connected 3-manifold and let $M:=W^{\circ}$. We distinguish the following cases:

- If at least one of the boundary components of $W$ has genus at least 2, then the finiteness criterion [9, p. 17] [14, Theorem 6.4] shows that $\|M\|^{\mathrm{lf}}=\infty$.

- If the boundary of $W$ consists only of spheres and tori, then we proceed as follows: In a first step, we fill in all spherical boundary components of $W$ by 3-balls and thus obtain an oriented compact connected 3-manifold $V$ all of whose boundary components are tori. In view of considerations on tame manifolds with amenable boundary [12] and glueing results for bounded cohomology [9] [2], we obtain that

$$
\|M\|^{\text {lf }}=\|W\|=\|V\| .
$$

By Kneser's prime decomposition theorem [1, Theorem 1.2.1] and the additivity of (relative) simplicial volume with respect to connected sums [2,9] in dimension 3, we may assume that $V$ is prime (i.e., admits no non-trivial decomposition as a connected sum). Moreover, because $\left\|S^{1} \times S^{2}\right\|=0$, we may even assume that $V$ is irreducible [1, p. 3].

By geometrisation [1, Theorem 1.7.6], then $V$ admits a decomposition along finitely many incompressible tori into Seifert fibred manifolds (which have trivial simplicial volume [23, Corollary 6.5.3]) and hyperbolic pieces $V_{1}, \ldots, V_{k}$. As the tori are incompressible, 
we can now again apply additivity [2,9] to conclude that

$$
\|V\|=\sum_{j=1}^{k}\left\|V_{j}\right\| .
$$

Let $j \in\{1, \ldots, k\}$. Then the boundary components of $V_{j}$ are $\pi_{1}$-injective tori (as the interior of $V_{j}$ admits a complete hyperbolic metric of finite volume) [4, Proposition D.3.18]. Let $S$ be a Seifert 3-manifold whose boundary is a $\pi_{1}$-injective torus (e.g., the knot complement of a non-trivial torus knot [21, Theorem 2] [17, Lemma 4.4]). Filling each boundary component of $V_{j}$ with a copy of $S$ results in an oriented closed connected 3-manifold $N_{j}$, which satisfies (again, by additivity)

$$
\left\|N_{j}\right\|=\left\|V_{j}\right\|+0=\left\|V_{j}\right\| .
$$

Therefore, the oriented closed connected 3-manifold $N:=N_{1} \# \ldots \# N_{k}$ satisfies

$$
\|N\|=\sum_{j=1}^{k}\left\|N_{j}\right\|=\sum_{j=1}^{k}\left\|V_{j}\right\|=\|V\| .
$$

In particular, $\|M\|^{\mathrm{lf}}=\|V\|=\|N\| \in \mathrm{SV}(3)$.

Funding Open Access funding enabled and organized by Projekt DEAL. This work was supported by the CRC 1085 Higher Invariants (Universität Regensburg, funded by the DFG).

Open Access This article is licensed under a Creative Commons Attribution 4.0 International License, which permits use, sharing, adaptation, distribution and reproduction in any medium or format, as long as you give appropriate credit to the original author(s) and the source, provide a link to the Creative Commons licence, and indicate if changes were made. The images or other third party material in this article are included in the article's Creative Commons licence, unless indicated otherwise in a credit line to the material. If material is not included in the article's Creative Commons licence and your intended use is not permitted by statutory regulation or exceeds the permitted use, you will need to obtain permission directly from the copyright holder. To view a copy of this licence, visit http://creativecommons.org/licenses/by/4.0/.

\section{References}

1. Aschenbrenner, M., Friedl, S., Wilton, H.: 3-manifold groups. EMS Series of Lectures in Mathematics. European Mathematical Society (EMS), Zürich (2015)

2. Bucher, M., Burger, M., Frigerio, R., Iozzi, A., Pagliantini, C., Pozzetti, M.B.: Isometric embeddings in bounded cohomology. J. Topol. Anal. 6(1), 1-25 (2014)

3. Bucher, M., Inkang, K., Sungwoon, K.: Proportionality principle for the simplicial volume of families of $\mathbb{Q}$-rank 1 locally symmetric spaces. Math. Z. 276(1-2), 153-172 (2014)

4. Benedetti, R., Petronio, C.: Lectures on Hyperbolic Geometry. Universitext. Springer, Berlin (1992)

5. Brown, M.: Locally flat imbeddings of topological manifolds. Ann. Math. 2(75), 331-341 (1962)

6. Connelly, R.: A new proof of Brown's collaring theorem. Proc. Am. Math. Soc. 27, 180-182 (1971)

7. Fujiwara, K., Manning, J.F.: Simplicial volume and fillings of hyperbolic manifolds. Algebr. Geom. Topol. 11(4), 2237-2264 (2011)

8. Frigerio, R., Moraschini, M.: Gromov's theory of multicomplexes with applications to bounded cohomology and simplicial volume. Mem. Amer. Math. Soc., to appear, 2018. arXiv:1808.07307 [math.GT]

9. Gromov, M.: Volume and bounded cohomology. Inst. Hautes Études Sci. Publ. Math., (56):5-99 (1983), 1982

10. Heuer, N., Löh, C.: The spectrum of simplicial volume. Invent. Math. 223, 103-148 (2021). https://doi. org/10.1007/s00222-020-00989-0

11. Heuer, N., Löh, C.: Transcendental simplicial volumes. Annales de l'Institut Fourier, 2020. to appear 
12. Kim, S., Kuessner, T.: Simplicial volume of compact manifolds with amenable boundary. J. Topol. Anal. 7(1), 23-46 (2015)

13. Kirby, R.C., Siebenmann, L.C.: On the triangulation of manifolds and the Hauptvermutung. Bull. Am. Math. Soc. 75, 742-749 (1969)

14. Löh, C.: Isomorphisms in $l^{1}$-homology. Münster J. Math. 1, 237-265 (2008)

15. Löh, C., Sauer, R.: Degree theorems and Lipschitz simplicial volume for nonpositively curved manifolds of finite volume. J. Topol. 2(1), 193-225 (2009)

16. Löh, C., Sauer, R.: Simplicial volume of Hilbert modular varieties. Comment. Math. Helv. 84(3), 457-470 (2009)

17. Lück, W.: $L^{2}$-Invariants: Theory and Applications to Geometry and $K$-Theory, volume 44 of Ergebnisse der Mathematik und ihrer Grenzgebiete. 3. Folge. A Series of Modern Surveys in Mathematics [Results in Mathematics and Related Areas. 3rd Series. A Series of Modern Surveys in Mathematics]. SpringerVerlag, Berlin, 2002

18. May, J.P.: A concise course in algebraic topology. Chicago Lectures in Mathematics. University of Chicago Press, Chicago, IL (1999)

19. Matsumoto, S., Morita, S.: Bounded cohomology of certain groups of homeomorphisms. Proc. Am. Math. Soc. 94(3), 539-544 (1985)

20. Moise, E.E.: Geometric topology in dimensions 2 and 3. Graduate Texts in Mathematics, vol. 47. Springer, New York (1977)

21. Moser, L.: Elementary surgery along a torus knot. Pac. J. Math. 38, 737-745 (1971)

22. Siebenmann, L.C.: On the homotopy type of compact topological manifolds. Bull. Am. Math. Soc. 74, 738-742 (1968)

23. Thurston, W.P.: Three-Dimensional Geometry and Topology. Vol. 1, volume 35 of Princeton Mathematical Series. Princeton University Press, Princeton, NJ, 1997. Edited by Silvio Levy

Publisher's Note Springer Nature remains neutral with regard to jurisdictional claims in published maps and institutional affiliations. 